\title{
"El enfoque estratégico en el pensamiento directivo del Administrador de Empresas"
}

\author{
Lic. Adm. Fernando MÁLAGA MÁLAGA ${ }^{19}$
}

\section{RESUMEN:}

En una perspectiva teórica dominante en la gestión estratégica, se debe considerar, ab initio, los enfoques de recursos de la organización. En este artículo, una visión teórica y analítica principal de estos enfoques se propone, como punto de partida, para la gestión de las capacidades estratégicas de la empresa. La capacidad de la empresa para enfocar sus recursos hacia el dominio de la dinámica en la toma de decisiones consistentes, se convierte en un tema central del enfoque estratégico de la planificación organizativa integrada.

El pensamiento de los connotados autores consultados en una compilación comentada, está expuesto, de manera algo tautológica. Acerca de los autores, he puesto de relieve -subrayando por mi cuenta- algunas ideas que pueden resultar interesantes. La referencia bibliográfica es sugerente para que el lector profundice lo que despierte su mayor interés.

PALABRAS CLAVE: Enfoque, Pensamiento, Estrategia, Fases.

\section{ABSTRACT:}

In a dominant theoretical perspective in strategic management, must be ab initio considered, the approaches to organizational resources. In this paper, a theoretical and main analytical vision of these approaches is proposed as a starting point for the management of the strategic capabilities of the firm. The thought of the renowned authors consulted is exposed in an annotated and commented compilation, somewhat tautological. About the authors, I have highlighted some ideas that might be interesting, underlining them on my own. The ability of the company to focus its resources toward mastery of dynamics in making consistent decisions becomes a central theme of strategic approach to integrated organizational planning.

KEYWORDS: Focusing, Thought, Strategy, Phases. 


\section{OBJETIVOS E IMPORTANCIA DE UN ENFOQUE}

El enfoque estratégico pasa por un dilema que hace a veces difícil escoger las soluciones correctas. Muchas de las llamadas 'soluciones del problema' no son, en realidad, otra cosa que forzar restricciones. Sin embargo, la primera etapa del pensamiento estratégico consiste en determinar con precisión el punto crítico de la situación o de las debilidades, valores, políticas y otros, en los distintos procesos operativos, para analizarlos desde el principio. Un interesante enfoque de las etapas del pensamiento estratégico, es el planteado por Kenichi Ohmae, definido en una estructura de pasos, que va de "la abstracción seguida del acercamiento, hacia un plan completo de mejoras:
a) Localización de fenómenos.
b) Agrupación de éstos.
c) Abstracción.
d) Determinación del enfoque.
e) Formulación provisional de las soluciones hipotéticas.
f) Validación o rechazo de las soluciones hipotéticas por medio de un análisis profundo.
g) Surgimiento de la conclusión.
h) Concreción de la forma de las conclusiones.
i) Proyecto del plan de acción.
j) Implementación por los gerentes de línea ${ }^{20}$

Los enfoques estratégicos pueden variar, dependiendo de las características propias de cada negocio en el que se mueve una empresa: comercial, industrial, de servicio, etc. Una visión moderna, al respecto, plantea Richard Whittington, clasificando los enfoques en cuatro tipos:

a) Clásico: es principalmente para empresas en industrias maduras, de uso intensivo del capital, que tienen poder de monopolio.

b) Evolucionista: adecuado para pequeñas empresas, empresas en sectores e industrias emergentes, y los conglomerados anglosajones.

c) Procesualista: para empresas con burocracias protegidas y las empresas basadas en conocimiento.

d) Sistémico: para empresas en países con modelo económico no anglosajón, empresas familiares y estatales (para-estatales).

\section{FILOSOFÍA FUNDACIONAL}

El enfoque estratégico basado en la filosofía fundacional se nutre de la visión y misión de los pioneros que establecieron la cultura empresarial para llevar adelante a su firma dentro de una configuración "misionera inicial". Tal fue la idea, por ejemplo, de constituirse en la primera empresa de producción de helados en el Perú, la que llevó a 
los hermanos D’Onofrio a fundar y mantener el liderazgo en su ramo, en casi un siglo de existencia, frente a varios emprendimientos similares, que fracasaron al competir.

"Las metas y valores personales dan forma, inevitablemente, a la estrategia de la organización, nos dice Stoner. Por ejemplo -continúa- Walt Disney concedia gran valor a la diversión familiar y concibió la idea de crear un 'parque mágico' que divirtiera y educara los niños y a sus padres. Su visión produjo Disneylandia, que abrió sus puertas en 1955. Aunque Disney murió en 1966, sus valores y visión han seguido dando forma a su empresa ${ }^{21}$. Los parques Disney en Europa, Japón y China, dan fe de ello.

Al formar una empresa peculiar, desde sus primeros años de existencia, la creatividad de cualquier persona produce inmediatamente una propuesta de diferenciación para establecer las raíces de su ideología fundacional. Con el espacio y tiempo histórico, esos ideales primigenios se irán contrastando con los logros alcanzados por la organización, más allá de sus fundadores, para determinar el carácter de su misión distintiva, fiel a su espíritu inicial, pero flexible y acorde con los tiempos vigentes.

\section{EL PENSAMIENTO ESTRATÉGICO}

En la etapa del pensamiento estratégico, lo primero que debe buscarse es el claro entendimiento del carácter particular de cada elemento en una coyuntura dada y después hacer el más completo uso posible del poder de nuestras capacidades intelectuales y racionales, en beneficio del proyecto común. Es muy importante, reestructurar esos elementos en la forma más ventajosa, pues los fenómenos y eventos del mundo real no siempre siguen una tendencia lineal. El medio más confiable para discriminar una situación por sus componentes y volverlos a ensamblar con el modelo deseado, no es la metodología gradual de los sistemas de análisis; más bien, es la herramienta fundamental del pensamiento no lineal: el cerebro humano.

\section{“Qué es el pensamiento estratégico y por qué es importante?”}

La interrogante es planteada por Morrisey, quien nos da su respuesta en dos planos:

1. "El pensamiento estratégico individual incluye la aplicación del juicio basado en la experiencia para determinar las direcciones futuras.

2. El pensamiento estratégico de empresa es la coordinación de mentes creativas dentro de una perspectiva común que le permita a un negocio avanzar hacia el futuro de una manera satisfactoria para todos "22.

De ahí surge el esclarecimiento que significa hacer un alto en la rutina diaria y dedicarle tiempo sólo al pensamiento, para lo cual casi nunca hay tiempo. El estratega, igual

21 Whittington, Richard: “¿Qué es la estrategia? ¿Realmente importa?

22 Stoner., James A.F, Freeman, R. Edward y Gilbert, Daniel R.: "Administración” 
que el buen gerente descrito por H. Edward Wrapp, "solamente puede aportar sus talentos especiales a un número limitado de asuntos... y en circunstancias normales se limitará a sí mismo a tres o cuatro objetivos fundamentales "23.

Pensar estratégicamente es superar los parámetros del tiempo en favor de una "tarea administrativa por objetivos y de autocontrol", a lo que Drucker llama con rigor, "la filosofia de la administración". Bajo este paraguas "cubridor" se cobija el pensamiento estratégico que "descansa en un concepto de la acción, el comportamiento y la motivación humanos... aplicable a todos los gerentes, sea cual fuere su nivel y su función, y a cualquier organización, grande o pequeña. Garantiza el desempeño convirtiendo las necesidades objetivas en metas personales. Y esto es auténtica libertad"24, de pensamiento y acción, añadiremos.

\section{FASES y CAMPOS DE ACCIÓN}

El sentido común nos debería decir que entre lo más importante para la proyección empresarial está saber cómo influyen en ella, las amplias corrientes de los cambios económicos y po-

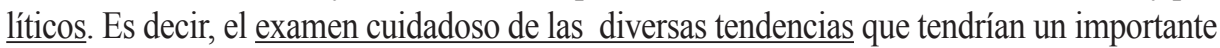
impacto en las estrategias de los negocios de los años venideros. Éstos son los factores influyentes del entorno, cuyos mayores alcances merecen un detenido tratado aparte.

Obviamente, el pensador estratégico hará bien en entender los antecedentes de estos factores, sus implicancias para la empresa y las formas en que deberán concebirse las estrategias luego de diagnosticar el ambiente en base a tomar en cuenta estos y otros indicadores. La siguiente fase pensante debe llevar al estratega a estimar los campos de acción más adecuados para orientar la aplicabilidad de los modelos 'macro' a la realidad 'micro' que son las empresas. Esta dirección puede dar lugar a diversas posiciones estratégicas como las que puntualmente sugiere Andrews, basándose en experiencias de las que considera "empresas reales:

\begin{tabular}{|l|}
\hline - Primero: \\
\hline $\begin{array}{l}\text { La conclusión de que la estrategia se gestiona en las empresas durante unos ciertos periodos } \\
\text { de tiempo, tal como (se) ha podido comprender por estudios hechos en situaciones reales. }\end{array}$ \\
\hline - Segundo: \\
\hline $\begin{array}{l}\text { Ya que la estrategia cambia con el transcurso del tiempo, por desgaste de los } \\
\text { supuestos en que se basa o por una rectificación consciente de la dirección, estos } \\
\text { enunciados probablemente no son definiciones de la estrategia en curso. }\end{array}$ \\
\hline - Tercero: \\
$\begin{array}{l}\text { Es tan persistente el punto de vista funcional o del especialista, que seguramente cada } \\
\text { uno de los diferentes directivos de estas empresas y analistas de los casos, redefiniría } \\
\text { la estrategia enunciada..., de manera que reflejase algún punto importante para él”.25. }\end{array}$ \\
\hline
\end{tabular}

23 Morrisey, George L.: "Pensamiento Estratégico"

24 Hamermesh, Richard G.: "Planeación Estratégica"

25 Drucker, Peter F.: "La Gerencia - Tareas, Responsabilidades y Prácticas" 


\section{Los campos de acción para el pensamiento estratégico sólo tienen, pues, como limitantes la creatividad y el espíritu innovador del propio estratega.}

Las fases de proyección, también se pueden estructurar de modo inverso, considerando las pruebas a las que se someterá la estrategia de negocios para medir su corrección. Henry Mintzberg ${ }^{26}$ distingue los siguientes criterios generales:

\begin{tabular}{|l|l|}
\hline “Consistencia: & La estrategia no deberá presentar metas ni políticas inconsistentes entre sí. \\
\hline Consonancia: & $\begin{array}{l}\text { La estrategia deberá representar una respuesta adaptativa al medio } \\
\text { ambiente externo, así como a los cambios relevantes que en él ocurren. }\end{array}$ \\
\hline Ventaja: & $\begin{array}{l}\text { La estrategia deberá facilitar la creación o la preservación de la } \\
\text { superioridad competitiva en el área elegida de actividades. }\end{array}$ \\
\hline Factibilidad: & $\begin{array}{l}\text { La estrategia no deberá agotar los recursos disponibles y } \\
\text { tampoco generar problemas irresolubles }{ }^{27} .\end{array}$ \\
\hline
\end{tabular}

\section{ENFOQUE DE LA CREACIÓN DE VALOR}

Es evidente que cuanto más alto sea el factor de la necesidad de cambio, se tendrá que hacer una mayor prospección previa del nivel de identificación del individuo con la organización y su lealtad hacia ella, para evaluar y reorientar las tendencias existentes. Este deslinde es importante porque tiene como fin optimizar los alcances presentes y desarrollar nuevos valores, para así, respectivamente, lograr reafirmar o atraer a los antiguos y nuevos miembros a compartir la escala de valores corporativos que encierran propuesta estratégica de la empresa.

La identificación también podría darse por la consistencia que se genera "entre los objetivos organizacionales y los valores del grupo administrativo"28. En este sentido, es necesario que el enfoque estratégico tenga mucha necesidad de imbuirse en reconocer cuál es la estructura ideológica valorativa del personal con la organización, para evitar conflictos entre las políticas de la empresa y los valores personales o sociales.

\section{CONCLUSIONES}

Aunque a veces, la planeación estratégica puede sonar muy teórica, la etapa inicial del pensamiento estratégico comprende enfocarse principalmente en el análisis situacional. La planificación estratégica por sí sola no puede resolver todos los problemas de las organizaciones, pero los procesos que intervienen para enfocarse en un Plan Estratégico pueden ser muy útiles en la identificación de algunas debilidades y oportunidades. Lo ideal es que el proceso de planificación estratégica sea cíclico, para que los temas críticos sean monitoreados y revisados con regularidad y la información clave que se recoja sea aplicable para la planificación de los nuevos objetivos.

26 Andrews, Kenneth R.: "El Concepto de Estrategia de la Empresa"

27 Mintzberg, Henry; Quinn, James Brian y Voyer, John: "El Proceso Estratégico".

28 Ibid. Mintzberg 
El análisis demuestra que la revisión de estos temas es necesaria, con el fin de elaborar una estrategia perfecta, concertando las diversas ideas de los fundadores que emprendieron el negocio, con las que pueden surgir desde los empleados de la organización. El aporte de los colaboradores, como co-creación estratégica, podría contribuir a cambiar por completo el futuro de la empresa y llevarla a un nivel muy alto.

Es frecuente considerar que el aspecto principal que conduce al éxito o fracaso de una organización es la estrategia, lo que es un error, porque la estrategia es inútil sin una gestión estratégica eficiente. Este concepto de implementación, es muy importante a tomar en cuenta en el plan, aún antes de la elaboración de una estrategia. El desarrollo de planes de acción (o planes de trabajo), es sus distintas fases, puede parecer detallado y tedioso en comparación con las fases anteriores de la planificación estratégica que a menudo parecen más creativas por su naturaleza.

Finalmente, hay que considerar que el enfoque en la creación del valor empresarial, debe tener como referente al plan matricial de la estrategia corporativa VBM (ValueBased Management). La gestión basada en el valor se dirige al éxito por las metas de desempeño que deben ser muy claras y adecuadamente alineadas con el objetivo final de crear valor. El enfoque aborda este problema frontalmente, se centra en mejores decisiones a todos los niveles en una empresa y va de la mano con la dirección, los funcionarios y los dependientes de la organización.

\section{REFERENCIAS BIBLIOGRÁFICAS}

1. Ohmae, Kenich. (1994). "La Mente del Estratega”- McGraw-Hill, Pág. 21.

2. Whittington, Richard. (2003). “QQué es la estrategia? ¿Realmente importa?", Madrid, Thomson.

3. Stoner., James A.F, Freeman, R. Edward y Gilbert, Daniel R. (1996). “Administración” - Prentice-Hall, Pág. 322.

4. Morrisey, George L. (1996). "Pensamiento Estratégico”- PrenticeHall, Pág. 2.

5. Hamermesh, Richard G. (1997) “Planeación Estratégica” - Limusa, Pág. 83.

6. Drucker, Peter F. “La Gerencia - Tareas, Responsabilidades y Prácticas”, Pág. 301.

7. Andrews, Kenneth R. (1977). "El Concepto de Estrategia de la Empresa”-EUNSA. Pág. 62.

8. Mintzberg, Henry; Quinn, James Brian y Voyer, John. (1997). "El Proceso Estratégico”- Págs. 81, 82.

9. Ibid. Mintzberg, Pág.82. 